\title{
Characteristic Analysis of longitudinal vibration Sucker Rod String With Variable Equivalent Stiffness
}

\author{
Jian LV ${ }^{1}$, Mingming Xing ${ }^{2}$ \\ ${ }^{1}$ School of Mechanical and Vehicle Engineering, Linyi University, Linyi 276000, China \\ ${ }^{2}$ School of Automation and Electrical Engineering, Linyi University, Linyi 276000,China
}

\begin{abstract}
Considering the influence of variable equivalent stiffness on system response, the equivalent stiffness is defined as a step function, and a mathematical model of nonlinear longitudinal vibration of sucker rod string (SRS) is built. The dynamic response under displacement and load force excitation is solved by fourth-order Runge-Kutta method with zero initial condition. The results show the steady-state responses under the displacement and load force excitation of different function forms are different. The response curves of both displacement and velocity under the displacement and load force excitation of cosine function form have larger fluctuation than it under the displacement and load force excitation of sine function form. Therefore, the characteristic analysis of SRS plays an important role in understanding the influence of the excitation form and sensitive parameters on steady response.
\end{abstract}

\section{Instruction}

The SRS is a slender rod of several kilometres, which transmits the movement from mechanical rotary motion to plunger linear motion [1-2]. And, the characteristic analysis of longitudinal vibration of SRS is very important for the parameters optimization of sucker rod pumping system (SRPS) [3]. Therefore, the dynamic simulation of SRS is studied by domestic and foreign scholars. After wave equation of Gibbs [4], an improved model of SRPS is built by Dale Russel Doty and S.D.Lekia [5-6]. Recently, I.N.Shardakov, Luan Guo-hua and M.M Xing etal [7-9] analyse boundary conditions effecting rod law of motion and correct the excitation forces of rod longitudinal vibration. However, the most of aforementioned approaches have not been studied sufficiently. Relevant references [9-10] shows that the equivalent stiffness is a function of time, when the influence of the helical buckling of SRS on the equivalent stiffness is taken into consideration, which affects the characteristic of longitudinal vibration of SRS. Therefore, with non-constant equivalent stiffness, the dynamic Characteristic of SRS with Variable Equivalent Stiffness is necessary to be analysed.

In this paper, the dynamic model and nonlinear second-order differential equations are built, and the entire equilibrium equation is given in section 2. In section 3, the system equations of nonlinear longitudinal vibration of SRS are solved by fourth-order Runge-Kutta method with zero intimal conditional. In section 4 , the dynamic responses under different function forms of load force excitation as well as the load force and displacement excitation are given, and the effects of different function forms of load force excitation as well as displacement excitation on the amplitude-frequency curves are analysed. In section 5, the significance of dynamic characteristic of SRS and conclusions are summarized.

\section{Dynamic model and nonlinear second-order differential equations}

In order to establish mechanical model of nonlinear longitudinal vibration for SRS, the fundamental assumptions are as follows: (1)The tubing string is anchored; (2)The viscous damping of liquid is concerned; (3) The tube- liquid column vibration is ignored; (4) Considering the deformation of helical buckling of SRS, the equivalent stiffness is a function of time.

Based on the assumptions, SRS is dispersed into a spring-mass-damper system and a mechanical model of nonlinear longitudinal vibration for SRS is established, in Fig.1. Fig.1 shows that SRS is a spring-mass-damper system with constant stiffness in $0 \sim \mathrm{Li}$ units and with non-constant stiffness in residual units. The direction from up-bottom is described as positive. The displacement, velocity and acceleration for polished rod are defined as $\mathrm{u} 0, \dot{u}_{0}, \ddot{u}_{0}$. Then a mathematical model of nonlinear longitudinal vibration of SRS is established, as follows. 


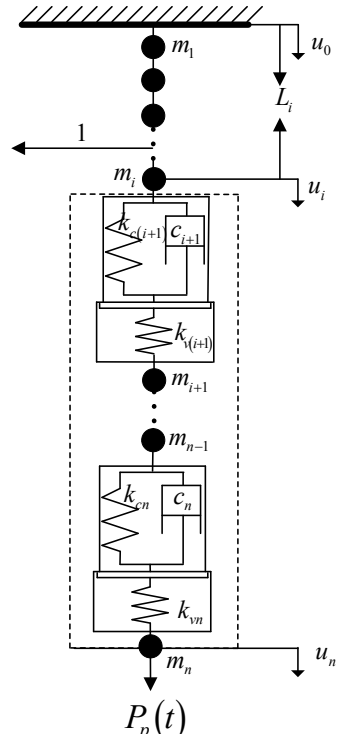

(a)

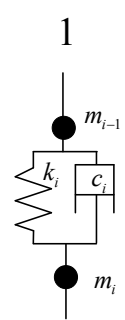

(b)
Figure 1. Mechanical model of nonlinear longitudinal vibration of SRS

$$
\begin{aligned}
& m_{1} \ddot{u}_{1}+k_{1}(t)\left(u_{1}-u_{0}\right)+k_{2}(t)\left(u_{1}-u_{2}\right)+c_{1}\left(\dot{u}_{1}-\dot{u}_{0}\right)+c_{2}\left(\dot{u}_{1}-\dot{u}_{2}\right)=0 \\
& m_{2} \ddot{u}_{2}+k_{2}(t)\left(u_{2}-u_{1}\right)+k_{3}(t)\left(u_{2}-u_{3}\right)+c_{2}\left(\dot{u}_{2}-\dot{u}_{1}\right)+c_{3}\left(\dot{u}_{2}-\dot{u}_{3}\right)=0
\end{aligned}
$$

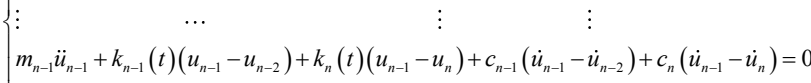

$$
\begin{aligned}
& m_{n} \ddot{u}_{n}+k_{n}(t)\left(u_{n}-u_{n-1}\right)+c_{n}\left(\dot{u}_{n}-\dot{u}_{n-1}\right)-P_{p}(t)=0
\end{aligned}
$$

Defining $\{\mathrm{u}\}=\{\mathrm{u} 1, \mathrm{u} 2, \ldots, \mathrm{un}-1$, un $\} \mathrm{T}$, a simplified mathematical model is given, as follows

$$
[M]\{\ddot{u}\}+[K]\{u\}+[C]\{\dot{u}\}=\{F\}
$$

Where,

$$
\begin{gathered}
m_{i}= \begin{cases}\frac{\rho_{r} g l_{0 i} \pi D_{i}^{2}}{4} & i=1,2,3 \cdots n-1 \\
\frac{\rho_{r} g l_{0 i} \pi D_{i}^{2}}{8} & i=n\end{cases} \\
c_{i}=\frac{12 \pi \mu m_{i}}{\rho_{r i} A_{r i}}\left(\frac{D_{r i}}{D_{t i}-D_{r i}}\right)\left[\left(0.20+0.39 \frac{D_{r i}}{D_{t i}}\right)+\frac{2.197 \times 10^{4}}{25}\left(\frac{D_{c i}}{D_{t i}}-0.381\right)^{2.57} \frac{D_{c i}{ }^{2}-D_{r i}{ }^{2}}{l_{0 i} D_{r i}}\right]
\end{gathered}
$$

$$
\begin{gathered}
\quad\left\{\begin{array}{l}
u_{0}=A_{0} \sin \left(\omega_{f} t\right) \\
P_{p}(t)=M_{0}+M_{1} \sin \left(\omega_{f} t\right)
\end{array}\right. \\
k_{x}=\left\{\begin{array}{l}
x=1,2, \cdots i \\
\frac{E_{r} A_{r i}}{l_{0 i}} \\
x=i+1, i+2, \cdots n \\
\operatorname{sgn}(\dot{u}) \varepsilon(\dot{u}) \frac{E_{r} A_{r i}}{l_{0 i}}+\operatorname{sgn}(-\dot{u}) \varepsilon(-\dot{u}) \frac{k_{c i} k_{v i}(t)}{k_{c i}+k_{v i}(t)}
\end{array}\right.
\end{gathered}
$$

Where mi is the element mass of SRS $(\mathrm{kg})$; $\rho$ r is the element density of SRS $(\mathrm{kg} / \mathrm{m} 3) ; \mathrm{Di}$ is the element diameter of SRS (m); 10i is the element length of SRS (m). $\mathrm{Er}$ is the Young's modulus of SRS $(\mathrm{Pa})$; Ari is the element cross-sectional area of SRS (m2); kvi is the variable equivalent stiffness $(\mathrm{N} / \mathrm{m}) ; \Delta \gamma \mathrm{vi}$ is the deformation of helical buckling section for SRS (m). $\mu$ is the kinematic viscosity of liquid (Pa.s), Dci is the outside diameter of sucker rod coupling $(\mathrm{m})$, Dti is the outside diameter of tube (m), Dri is the element diameter of SRS (m).

For the researches on the deformation of helical buckling for SRS, Lubinski, Xiaojun, Mitchell and Guohua give theoretical analysis method and experiment [11-13]. And the damping coefficient is derived by Shimin, and the equation is expressed [2]. The main aim of this paper is to analyse the characteristic of SRPS. Therefore, the research on deformation of helical buckling for SRS is reported in Refs. [11-13] and need not be repeated here.

\section{Solutions}

The system equations of nonlinear longitudinal vibration of SRS are solved by fourth-order Runge-Kutta method. Therefore, the numerical model of nonlinear longitudinal vibration of SRS is obtained.

$$
\left\{\begin{array}{l}
d y(1)=y(2) \\
d y(2)=\frac{1}{m_{1}}\left\{k_{1}(t) u_{0}-\left[k_{1}(t)+k_{2}(t)\right] u_{1}+k_{2}(t) u_{2}+c_{1} \dot{u}_{0}-\left(c_{1}+c_{2}\right) \dot{u}_{1}+c_{2} \dot{u}_{2}\right\} \\
d y(3)=y(4) \\
d y(4)=\frac{1}{m_{2}}\left\{k_{2}(t) u_{1}-\left[k_{2}(t)+k_{3}(t)\right] u_{2}+k_{3}(t) u_{3}+c_{2} \dot{u}_{1}-\left(c_{2}+c_{3}\right) \dot{u}_{2}+c_{3} \dot{u}_{3}\right\} \\
\quad \ldots \\
d y(2 n-3)=y(2 n-2) \\
\quad \cdots
\end{array}\right.
$$

In addition, for the first calculation, the initial conditions are the same for all the plots as given below

$$
y(j)=0, \quad j=1,2,3, \cdots, 2 n
$$

\section{Displacement and velocity response}

The basic parameters are as follows: the diameter of SRS Dri $=0.022 \mathrm{~m}$, the length of SRS Lr $=1000 \mathrm{~m}$, the Young's modulus of the rod $\mathrm{Er}=2.1 \times 1011 \mathrm{~Pa}$, the density of the $\operatorname{rod} \rho \mathrm{r}=7850 \mathrm{~kg} / \mathrm{m} 3$, the deformation length of helical buckling section $\Delta \gamma \mathrm{vi}=10 \mathrm{i}$, the load parameter $\mathrm{M} 0=1000 \mathrm{~N}$ and $\mathrm{M} 1=9000 \mathrm{~N}$, the displacement parameter $\mathrm{A} 0=2 \mathrm{~m}$, the excitation frequency $\omega \mathrm{f}=1 \mathrm{rad} / \mathrm{s}$.

\subsection{Load excitation}

When the load force is a cosine function and the amplitude of load force is not changed, Fig.2 gives the the steady-state response of displacement and velocity at the bottom of SRS under load force of cosine function form. In addition, when the load force is a sine function and the amplitude of load force is not changed, Fig.3 gives the steady-state response of both displacement and velocity at the bottom of SRS under load force of sine function form. In the details, when the load force is a cosine function, the amplitude of displacement response is bigger than the amplitude under load force of sine function form, and the velocity response of SRS has larger fluctuation. In 
addition, when initial value of load force starts from zero, the amplitudes of displacement and velocity response will be smaller.

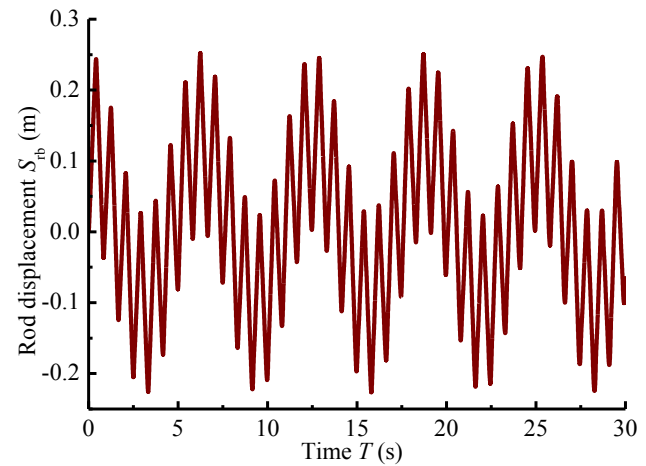

(a) Displacement of the bottom of SRS

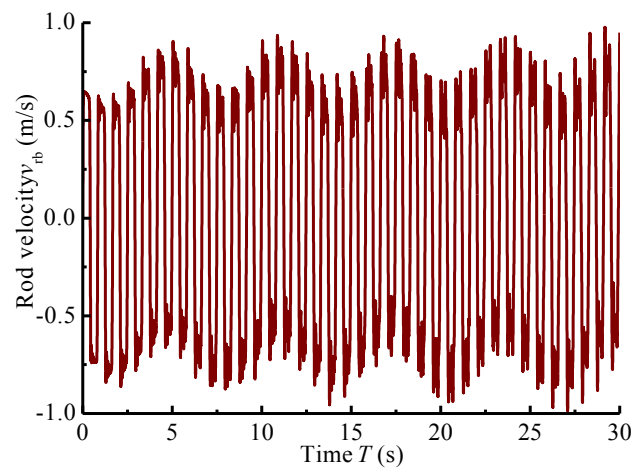

(b) Velocity of the bottom of SRS

Figure 2. The steady-state response of SRS under load force of cosine function form

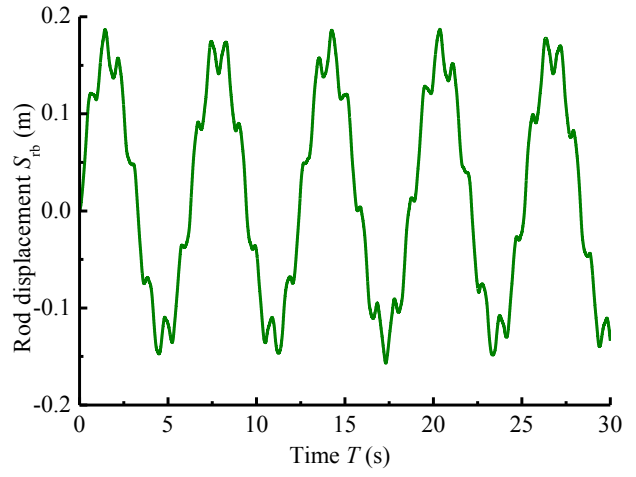

(a) Displacement of the bottom of SRS

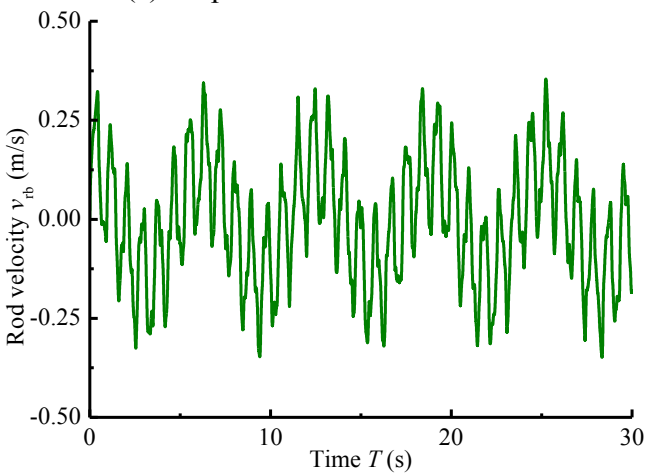

(b) Velocity of the bottom of SRS

Figure 3. The steady-state response of SRS under load force of sine function form

\subsection{Load force and displacement excitation}

When the load force is a cosine function and the displacement is a cosine or sine function, Fig.4 gives the steady-state response of both displacement and velocity at the bottom of SRS under load force of cosine function form. In addition, the load force is a sine function and the displacement is a cosine or sine function, Fig.5 gives the steady state response of both displacement and velocity at the bottom of SRS under load force of sine function form. According to the Figures, when the displacement excitation is a sine function, the amplitude response of both displacement and velocity are smaller than the response under displacement excitation of cosine function form in Fig.4. And the same phenomenon happens under load force of sine function form in Fig.5. Comparing Fig.4 with Fig.5, the amplitude of displacement response under load force of sine function form is bigger than the response under load force of cosine function form while the system is subjected to both load force and displacement excitation.

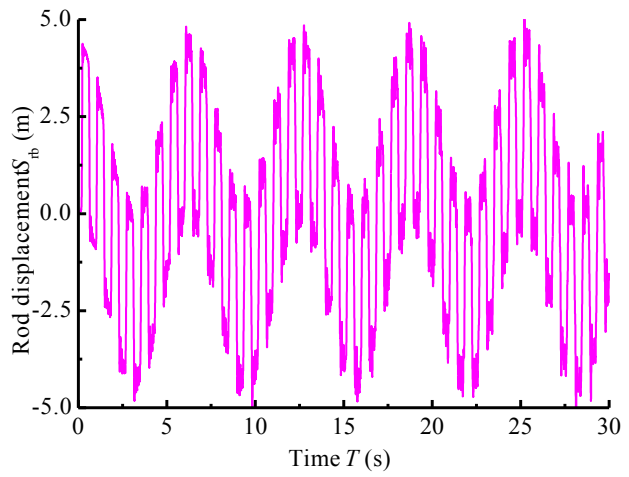

(a) Displacement of the bottom of SRS under displacement excitation of cosine function form

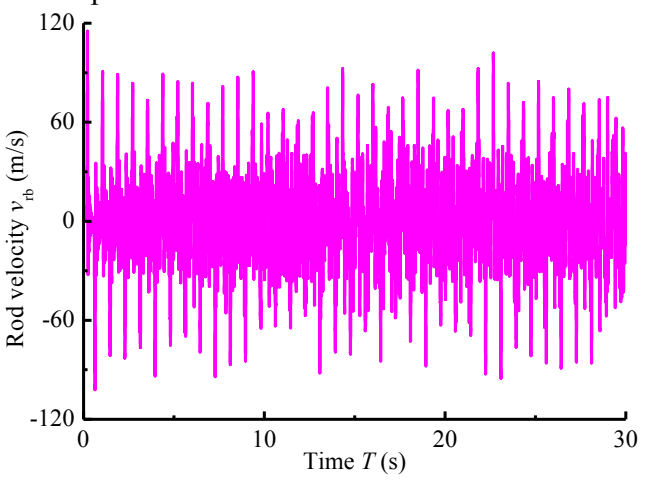

(b) Velocity of the bottom of SRS under displacement excitation of cosine function form

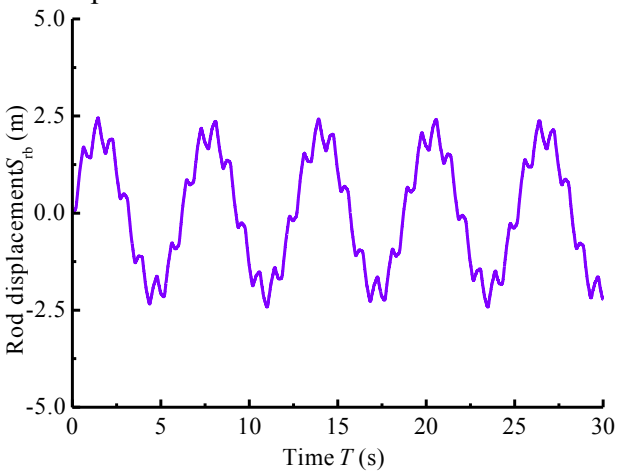


(c) Displacement of the bottom of SRS under displacement excitation of sine function form

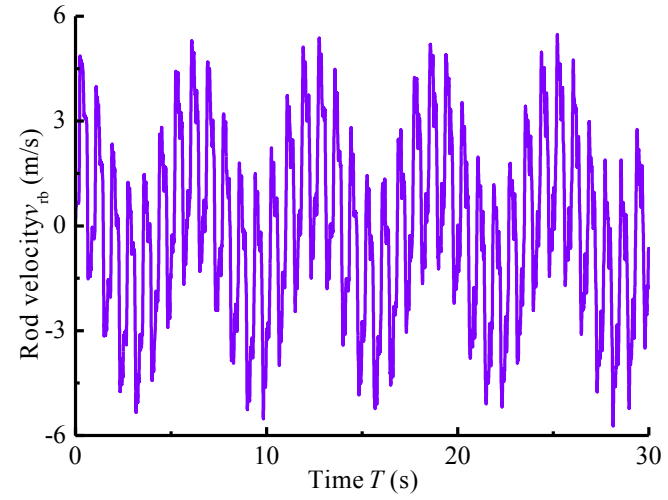

(d) Velocity of the bottom of SRS under displacement excitation of sine function form

Figure 4. The steady-state response of SRS under load force of cosine function form

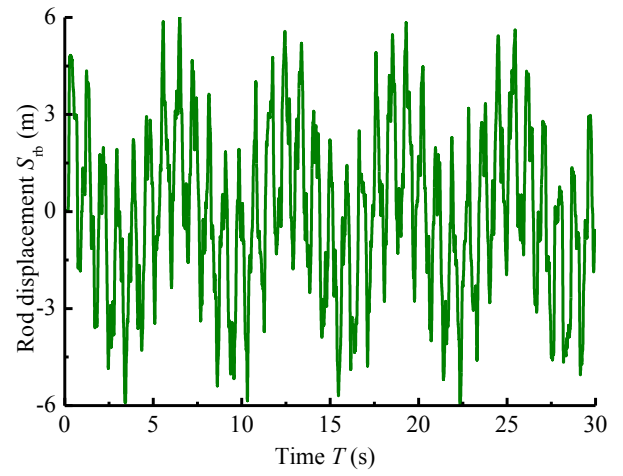

(a) Displacement of the bottom of SRS under displacement excitation of cosine function form

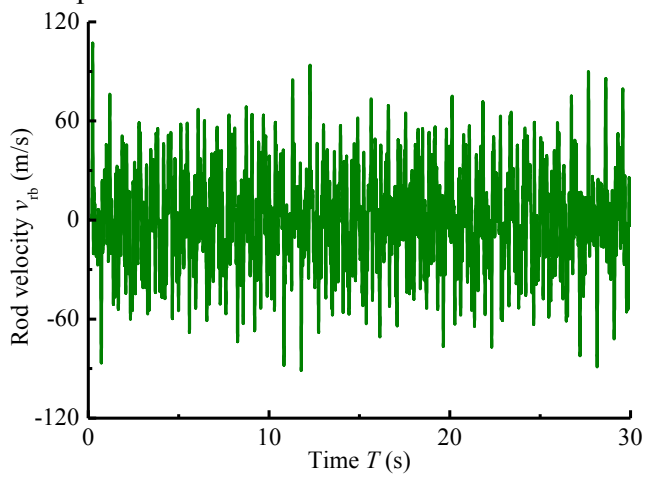

(b) Velocity of the bottom of SRS under displacement excitation of cosine function form

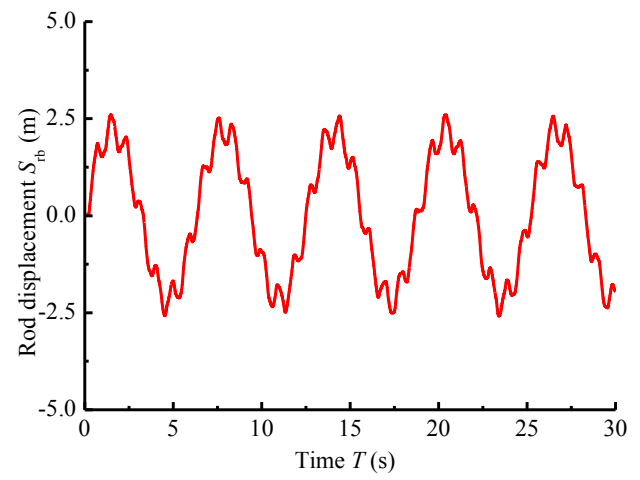

(c) Displacement of the bottom of SRS under displacement excitation of sine function form

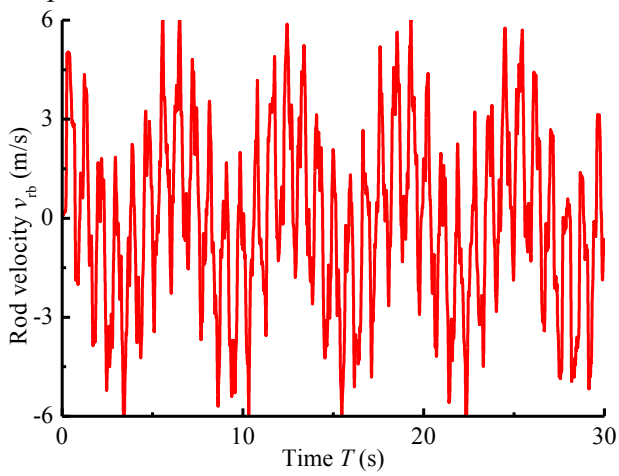

(d) Velocity of the bottom of SRS under displacement excitation of sine function form

Figure 5. The steady-state response of SRS under load force of sine function form

\section{Conclusions}

With the influence of the variable equivalent stiffness on steady state response, the sucker rod is dispersed into a spring-mass-damper mechanical system and a mathematic model of nonlinear longitudinal vibration of SRS is built. The dynamic response under displacement and load force excitation is solved by fourth-order Runge-Kutta method with zero initial condition.

The steady state responses under the displacement and load force excitation of different function forms are different. In the detail, the response curves of both displacement and velocity under the displacement and load force excitation of cosine function form have larger fluctuation than it under the displacement and load force excitation of sine function form. Therefore, the dynamic analysis of Sucker Rod String with variable equivalent stiffness plays an important role in understanding the influences of the excitation form and sensitive parameters on characteristic of SRS, which has important academic and practical significance for optimization of the performance parameters.

\section{Acknowledgments}

This research was financially supported by the Scientific Research Starting Foundation for Linyi University (Grant NO.LYDX2016BS032).

\section{References}

1. Xing M.M, Dong S.M. An improved longitudinal vibration model and dynamic characteristic of sucker rod string [J]. Journal of Vibroengineering, 16(7),3432-3448(2014).

2. Shimin Dong, Computer simulation of dynamic parameters of rod pumping system optimization[M]. Petroleum Industry Press, Beijing(2003).

3. Xing M.M, Dong S.M. A new simulation model of beam pumping system applied in energy saving and 
resource consumption reduction[J]. SPE Production \& Operations, 30(2):130-140(2015).

4. S.G. Gibbs, Predicting the behavior of sucker rod pumping systems, SPE Journal Paper 588-PA(1963).

5. S.G.Gibbs. and Neely, A.B, Computer Diagnosis of Down-Hole Conditions in Sucker Rod Pumping Wells, SPE Journal Paper, 1165-PA(1966).

6. S.G.Gibbs, A Review of Methods for Design and Analysis of Rod Pumping Installations, SPE Journal Paper, 9980-PA(1982).

7. I.N. Shardakov, I.N. Wasserman, Numerical modelling of longitudinal vibrations of a sucker rod string, Journal of Sound and Vibration 329(3, 1), 317-327 (2010).

8. DaCunha and Gibbs, Modeling a Finite-Length Sucker Rod Using the Semi-Infinite Wave Equation and a Proof to Gibbs' Conjecture, SPE Journal Paper, 108762-PA(2009).
9. Xing M.M. Response analysis of longitudinal vibration of sucker rod string considering rod buckling $[\mathrm{J}]$, Advances in Engineering Software, 99:49-58(2016).

10. S.A.M. Ghannadpour, B. Mohammadi, J. Fazilati, Bending, buckling and vibration problems of nonlocal Euler beams using Ritz method, Composite Structures 96, 584-589 (2013).

11. He, Xiaojun, Kyllingstad, Age, Helical Buckling and Lock-Up Conditions for Coiled Tubing in Curved Wells, SPE Journal Paper, 25370-PA (1995).

12. R.F. Mitchell, Buckling Behavior of Well Tubing: The Packer Effect, SPE Journal Paper, 9264-PA(1982).

13. Gao Guohua, Shell, and Stefan Miska, Effects of Friction on Post-Buckling Behavior and Axial Load Transfer in a Horizontal Well, SPE Journal Paper 120084-PA(2010). 